\title{
BEAM PHYSICS FOR THE 12 GEV CEBAF UPGRADE PROJECT*
}

\author{
J. Benesch, A. Bogacz, Y. Chao, A. Freyberger, J. Grames, L. Harwood, R. Kazimi, G. A. Krafft, \\ L. Merminga ${ }^{\#}$, M. Spata, M. Tiefenback, M. Wiseman, B. Yunn, Y. Zhang, \\ Jefferson Lab, Newport News, VA 23606, U.S.A.
}

\begin{abstract}
Beam physics aspects of the $12 \mathrm{GeV}$ Upgrade of CEBAF are presented. The CEBAF Upgrade to $12 \mathrm{GeV}$ is achieved via 5.5 recirculations through the linacs, and the installation of 10 new high-gradient cryomodules. A new experimental hall, Hall D, is envisioned at the end of the North Linac. Simulation results for a straight-ahead and a recirculated injector are summarized and compared. Beam transport designs are discussed and evaluated with respect to matching and beam breakup (BBU) optimization. Effects of synchrotron radiation excitation on the beam properties are calculated. BBU simulations and derived specifications for the damping of higher order modes of the new 7-cell cavities are presented. The energies that provide longitudinal polarization in multiple experimental halls simultaneously are calculated. Finally, detailed optics of the Hall D transport line has been obtained.
\end{abstract}

\section{INTRODUCTION}

A strong scientific case for the upgrade of the Continuous Electron Beam Accelerator Facility (CEBAF) at Jefferson Lab to $12 \mathrm{GeV}$ has been presented [1]. Such a facility will enable profound contributions to the study of hadronic matter. In particular, the following main areas will be explored: 1) The experimental study of gluonic excitations in order to understand the fundamentally new dynamics of quark confinement. 2) The determination of the quark and gluon wavefunctions of the nuclear building blocks. 3) Exploring the limits of our understanding of atomic nuclei. 4) Tests of the Standard Model and the determination of fundamental parameters of the model. The $12 \mathrm{GeV}$ Upgrade of CEBAF has received CD-0 Mission Needs statement and the development of the conceptual design report is presently in full swing. For the upgrade, a new experimental hall, Hall D, will be built at the end of the North Linac. In this hall collimated beams of linearly polarized photons at $8-9 \mathrm{GeV}$, produced by coherent bremsstrahlung from $12 \mathrm{GeV}$ electrons, will be used for experiments. The CEBAF Upgrade to $12 \mathrm{GeV}$ is achieved via 5.5 recirculations through the linacs, and the installation of 10 new high-gradient cryomodules [2]. The key parameters of the CEBAF upgrade are summarized in Table 1, and a schematic illustration of the project is shown in Figure 1. Beam physics studies in support of the upgrade are being carried out in the areas of injector design, beam transport, Higher Order Mode (HOM) damping specifications for the new cryomodules, polarization and Hall D transport line design.

\footnotetext{
* Work supported by DOE Contract DE-AC05-84ER40150

\#merminga@jlab.org
}

Table 1: 12 GeV CEBAF Upgrade key parameters

\begin{tabular}{|l|l|}
\hline Energy to Halls D /A,B,C & $12 \mathrm{GeV} / 11 \mathrm{GeV}$ \\
\hline $\begin{array}{l}\text { Number of passes for } \\
\text { Halls D / A,B,C }\end{array}$ & 5.5 (add a tenth arc) / 5 \\
\hline Duty Factor & $\mathrm{CW}$ \\
\hline $\begin{array}{l}\text { Max. Current to Halls } \\
\text { A,C / B,D }\end{array}$ & $85 \mu \mathrm{A} / 5 \mu \mathrm{A}$ \\
\hline New Cryomodules & $10(5$ per linac $)$ \\
\hline $\begin{array}{l}\text { Central Helium Liquefier } \\
\text { Upgrade }\end{array}$ & $\begin{array}{l}9 \mathrm{~kW} \\
(\sim 2 x \text { present capacity })\end{array}$ \\
\hline
\end{tabular}

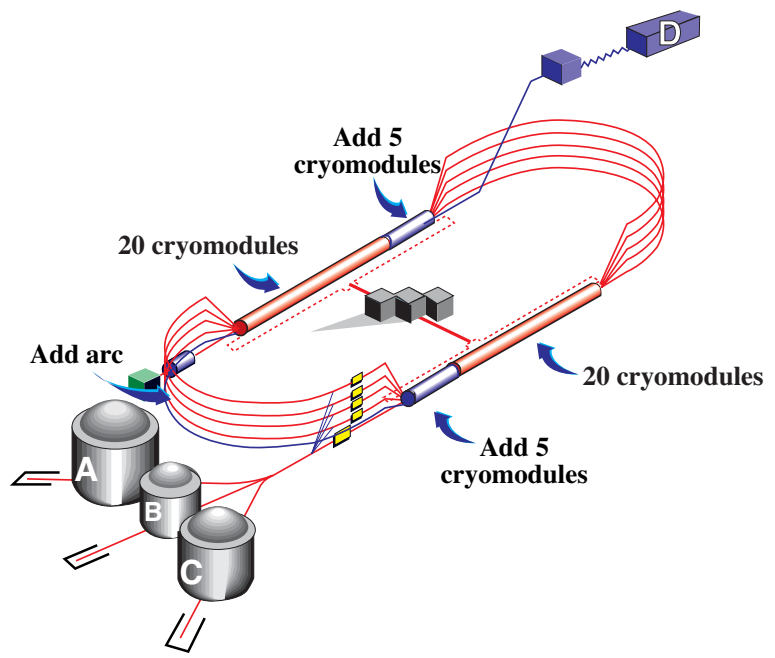

Figure 1: Schematic illustration of the CEBAF $12 \mathrm{GeV}$ Upgrade.

\section{INJECTOR}

The CEBAF $12 \mathrm{GeV}$ upgrade requires Injector energy of $123 \mathrm{MeV}$, an increase of $84 \%$ from the present configuration. This is necessary in order to maintain the existing geometry in spreaders and recombiners as energy gain is raised to $1090 \mathrm{MeV}$ per linac. We have two designs that achieve this goal [3]. One requires an upgrade to the existing accelerating modules and the other requires recirculation with lower capital cost (Fig. 2). We did a comparison of the two designs using computer modeling to ensure that both designs are technically viable. The single pass design performed very much the same as the existing lower energy injector design. The recirculated design was more complicated but the final beam parameters were within specifications. Sensitivity 
studies showed that for the recirculated design the final bunch length and energy spread are very sensitive to 5 $\mathrm{MeV}$ energy fluctuations that could come from RF phase or amplitude fluctuations. This was recognized to be due to large $\mathrm{M}_{56}$ in the recirculation line. We are studying changes to the design that can potentially reduce the $\mathrm{M}_{56}$ value in the recirculated injector. We are also assessing the operability of the recirculation option.

\section{BEAM TRANSPORT ISSUES}

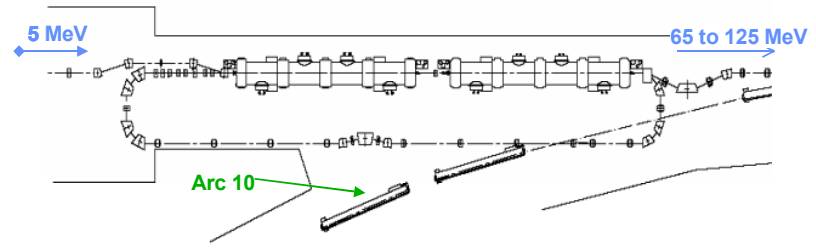

Figure 2. Recirculated Injector Layout

\section{Linac Energy Profile}

The $12 \mathrm{GeV}$ CEBAF linacs maintain the original design of a $120^{\circ}$ FODO lattice with constant focal length for the first pass. Distribution of the five new $98 \mathrm{MeV}$ cryomodules in each of the North and South Linacs, was dictated by maximum containment of betatron propagation through multiple passes in the North Linac, and minimal installation overhead in the South Linac. To arrive at this conclusion, a thorough parameter search and optimization program was executed, in order to answer questions regarding impact on machine operability as a function of not only arbitrary North Linac momentum profile, but also phase advance per cell and other tuning quad strengths. The analysis indicated the optimally achievable peak beam envelope in both planes for all 6 passes, and for the entire East end (Spreader, Linac, Recombiner) for each combination of tuning quad combination, under the following configuration options: a) New $100 \mathrm{MeV}$ cryo-modules located anywhere, including all in the front, all at the end, split in different ways between the front and end, and in the middle sections. b) Phase advance per cell at $120^{\circ}$ or $150^{\circ}$. The same program can be applied to any other phase advances. c) $1 \mathrm{~L} 28$ quad set to various strengths (e.g., $60 \%, 100 \%$, or $150 \%$ of nominal). The program gives the best achievable overall beam envelope for all configurations while allowing the freedom to use quads 1L00 and 1L01 as free parameters. More than a dozen scenarios corresponding to various combinations of the above options were studied and are summarized in [4]. Cost/benefit analysis of the options is going on. Were cost not a consideration, then the preference would be to place all new cryomodules at the low-energy end of the linac.

\section{Synchrotron Radiation Effects}

Synchrotron radiation-induced growth in emittance and energy spread results in large beam sizes at $12 \mathrm{GeV}$ and must be taken into account in the magnet aperture and field linearity specifications. Calculations of the effect with the present optics for passes 1 through 5, were carried out using the optics program Optim and are shown in Table 2, where all values are rms quantities. It should be noted that these emittances and energy spread meet the needs for the envisioned $12 \mathrm{GeV}$ research program.

Table 2: Transverse emittance and energy spread [rms]

\begin{tabular}{|c|c|c|c|}
\hline Area & $\begin{array}{c}\delta \mathrm{p} / \mathrm{p} \\
{\left[\mathrm{x} 10^{-3}\right]}\end{array}$ & $\begin{array}{c}\varepsilon_{\mathrm{x}} \\
{[\mathrm{mm}]}\end{array}$ & $\begin{array}{c}\varepsilon_{\mathrm{y}} \\
{[\mathrm{mm}]}\end{array}$ \\
\hline Chicane & 0.200 & $4.17 \mathrm{e}-6$ & $4.17 \mathrm{e}-6$ \\
\hline Arc 1 & 0.200 & $4.34 \mathrm{e}-7$ & $4.34 \mathrm{e}-7$ \\
\hline Arc 2 & 0.106 & $3.13 \mathrm{e}-7$ & $2.63 \mathrm{e}-7$ \\
\hline Arc 3 & 0.078 & $2.89 \mathrm{e}-7$ & $2.83 \mathrm{e}-7$ \\
\hline Arc 4 & 0.069 & $3.15 \mathrm{e}-7$ & $4.15 \mathrm{e}-7$ \\
\hline Arc 5 & 0.074 & $5.81 \mathrm{e}-7$ & $4.81 \mathrm{e}-7$ \\
\hline Arc 6 & 0.097 & $1.41 \mathrm{e}-6$ & $6.44 \mathrm{e}-7$ \\
\hline Arc 7 & 0.110 & $2.21 \mathrm{e}-6$ & $7.03 \mathrm{e}-7$ \\
\hline Arc 8 & 0.140 & $3.58 \mathrm{e}-6$ & $1.03 \mathrm{e}-6$ \\
\hline Arc 9 & 0.178 & $6.67 \mathrm{e}-6$ & $1.12 \mathrm{e}-6$ \\
\hline Arc 10 & 0.213 & $9.43 \mathrm{e}-6$ & $1.91 \mathrm{e}-6$ \\
\hline
\end{tabular}

\section{Spreaders and Recombiners}

The East Spreader and Recombiner have undergone extensive modification from the existing $6 \mathrm{GeV}$ CEBAF design to handle the increased beam energy and an additional 5.5-pass beamline out of the North Linac. The main deviations of the $12 \mathrm{GeV}$ east spreader from the original design occur in the separation scheme, where new septa magnets are introduced to perform staged separation, and in the dipoles which have to be either modified or increased in length to handle the higher energy beam. The east recombiner is a mirror image of the east spreader without the sixth pass. The additional sixth pass through the North Linac necessitates a new arc, Arc 10, with matching changes to the west spreader/recombiner. Arc 10 is composed of four superperiods analogous to the lower arcs, providing secondorder achromaticity and linear isochronicity.

\section{BEAM BREAKUP}

A detailed simulation study was carried out to determine the HOM damping requirement for the new 7cell CEBAF cavity needed to ensure the beam is inherently stable against multipass BBU instability. The study concludes that all deflecting (dipole) HOMs of the new 7-cell cavity should not exceed the following upper limit of $Q$ value

$$
Q=6.2 \times 10^{8} /(R / Q)_{T}
$$


where the HOM coupling strength is $(R / Q)_{T}=R / Q /(\mathrm{ka})^{2}$, $k$ is the wave number, and $a$ is the offset at which $R / Q$ is measured. This requirement guarantees stable beam operation of the CEBAF $12 \mathrm{GeV}$ upgraded machine with design currents [5].

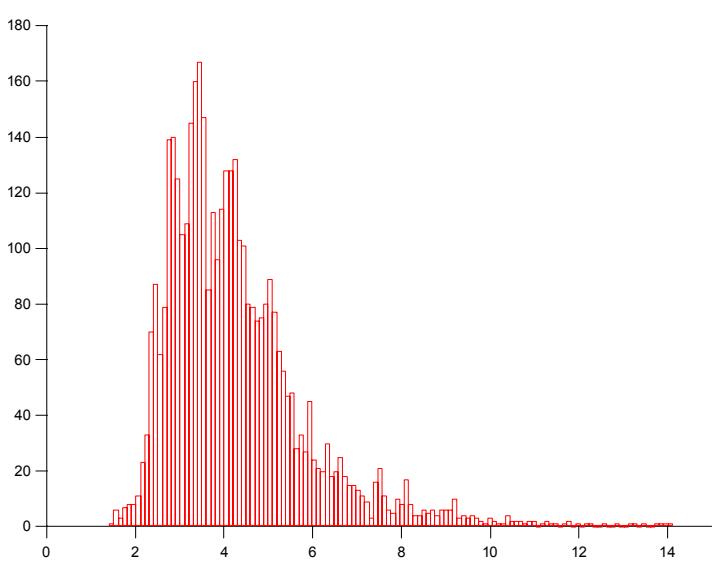

Figure 3: BBU threshold distribution (4000 sample machines) for the $1874 \mathrm{MHz}$ dipole mode with $\mathrm{Q}=$ $10^{6}$. Horizontal axis is the BBU threshold current in $\mathrm{mA}$ and vertical axis is the number of occurrences.

\section{POLARIZATION}

The majority of nuclear physics experiments approved at CEBAF require a highly polarized $(>75 \%)$ electron beam. Because the electron spins precess while traversing the accelerator only specific final beam energies, sometimes referred to as "magic energies" allow the beam polarization to be longitudinal in two experimental halls simultaneously, maximizing the experiment efficiency. The upgrade of CEBAF to $12 \mathrm{GeV}$ will augment the present capability, providing more than 500 combinations of simultaneous longitudinal beam polarization to any two of Halls A, B or C in the energy range 6 to $12 \mathrm{GeV}[6]$.

\section{HALL D OPTICS}

A lattice has been designed to separate sixth-pass beam at the end of the North Linac and take it to Hall D [7]. The beam line slopes upward at $10^{\circ}$ changing the beam height by $5.2 \mathrm{~m}$ finally ending up $1.25 \mathrm{~m}$ below grade. Three quad triplets and one dispersion correction quad are used to compensate for the incoming dispersion in the ramp. The large incoming horizontal emittance and the need to focus the virtual beam $75 \mathrm{~m}$ from the radiator to maximize photon polarization require the large increase in horizontal beta function in the final quadruplet. The lattice is shown in Fig. 4.



Figure 4. Hall D transport lattice: Beta functions (horizontal is red, vertical is green) and dispersion (black). Vertical scale is $[0,500] \mathrm{m}$ for beta functions (left axis), and $[-2,2] \mathrm{m}$ for dispersion (right axis). Horizontal scale is $[0,320] \mathrm{m}$.

\section{CONCLUSIONS}

Beam Physics issues for the $12 \mathrm{GeV}$ CEBAF Upgrade have been identified. A number of these issues have been resolved. More detailed studies, including particle tracking, are underway.

\section{REFERENCES}

[1] "Preconceptual Design Report (pCDR) for the Science and Experimental Equipment for the $12 \mathrm{GeV}$ Upgrade of CEBAF," http://www.jlab.org/div_dept/physics_division/pCDR public/pCDR_final/pCDR final.pdf

[2] L. Harwood, "JLab 12-GeV Upgrade," PAC'05, Knoxville, TN, May 2005, http://www.jacow.org.

[3] R. Kazimi, et al., "Injector Options for $12 \mathrm{GeV}$ CEBAF Upgrade," PAC'05, Knoxville, TN, May 2005, http://www.jacow.org.

[4] Y.-C. Chao, "Improved Optimization of North Linac Momentum Profile for CEBAF $12 \mathrm{GeV}$ Upgrade," JLAB-TN-05-004, 2005.

[5] B. Yunn, "HOM Damping Requirements for $12 \mathrm{GeV}$ Upgrade," JLAB-TN-04-035, 2004.

[6] J. Grames, "Magic Energies for the $12 \mathrm{GeV}$ Upgrade," JLAB-TN-04-42, 2004.

[7] J. Benesch, "Hall D Optics Design," JLAB-TN-03027, 2003. 